\title{
Presenting Neuropsychological Testing Profile of Autopsy-Confirmed Frontotemporal Lobar Degeneration
}

\author{
Hiroshi Yoshizawa ${ }^{a, e}$ Jean Paul G. Vonsattela, b Lawrence S. Honig ${ }^{a, c, d}$ \\ ${ }^{a}$ Taub Institute for Research on Alzheimer's Disease and the Aging Brain, ${ }^{b}$ Department of \\ Pathology and Cell Biology, ${ }^{c}$ Gertrude H. Sergievsky Center, and d Department of Neurology, \\ Columbia University College of Physicians and Surgeons, New York, N.Y., USA; e Department of \\ Neurology, Neurological Institute, Tokyo Women's Medical University, Tokyo, Japan
}

\section{Key Words}

Frontotemporal lobar degeneration · Frontotemporal dementia - Alzheimer's disease · Mild cognitive impairment · Neuropsychological assessment

\begin{abstract}
Aims: We aimed to investigate how neuropsychological test measures at presentation might differentiate frontotemporal lobar degeneration (FTLD) from Alzheimer's disease (AD). Methods: We compared autopsy-confirmed FTLD and definite AD with Clinical Dementia Rating $\leq 1$. Factor scores and $t$ values of each neuropsychological test measure were compared between FTLD and AD patients. Logistic regression analyses were applied to identify independent predictors within test measures for the differentiation of FTLD from AD. Results: Factor analyses showed that the memory domain was more severely impaired in AD than in FTLD, whereas the language and attention domains were more severely impaired in FTLD than in AD. Multiple logistic regression analysis showed that Letter Fluency, Boston Naming Test and delayed memory recall remained independent predictors of FTLD compared to AD. However, test measures did not discriminate between FTLD-tau and FTLD-ubiquitin. Conclusion: We confirm that memory and language function tests discriminate between FTLD and AD.
\end{abstract}

(c) 2013 S. Karger AG, Basel

\section{Introduction}

The clinical diagnosis of frontotemporal lobar degeneration (FTLD) in the early stages remains challenging. Although biomarkers from neuroimaging studies such as magnetic resonance imaging [1] and positron emission tomography [2] or from cerebrospinal fluid such as 
Yoshizawa et al.: Presenting Neuropsychological Testing Profile of Autopsy-Confirmed Frontotemporal Lobar Degeneration

tau and beta amyloid [3] may help distinguish FTLD patients from patients with Alzheimer's disease (AD), often neuropsychological testing is used for diagnosis in the clinical setting. However, it is less clear whether neuropsychological testing can discriminate FTLD from AD, especially in the early stages of dementia [4]. A meta-analytic review has suggested that the overlap in the neuropsychological performance of FTLD and AD patients can cause difficulties in differentiation of FTLD from AD [5]. While many authors have reported that the memory function of FTLD patients is relatively preserved compared with that of AD patients, detailed profiles of memory function have been less consistent [6, 7]. Finally, it has not been established how much cognitive testing can help in discriminating between the different pathologies underlying FTLD, which principally include FTLD-tau and FTLD-TAR DNA-binding protein of $43 \mathrm{kDa}$ (FTLD-TDP-43) [8-11].

In the present study, we investigated the neuropsychological testing features at presentation to our center of FTLD patients who had Clinical Dementia Rating (CDR) [12] $\leq 1$ and then ultimately had autopsy-confirmed FTLD. The objectives of this study were (1) to assess the differences in cognitive profiles between FTLD and AD at the early stage, (2) to investigate how the diagnostic accuracy for differentiation of FTLD from AD using neuropsychological measures can be improved, and (3) to delineate the neuropsychological features of different pathological bases of FTLD.

\section{Subjects and Methods}

Case Selection

Cases from the autopsy cohort of the Alzheimer's Disease Research Center (ADRC) at Columbia University were selected for this study if they met the primary neuropathological diagnosis of FTLD-tau, FTLD-ubiquitin/TDP-43-positive inclusions (FTLD-U) or definite AD and presented with CDR $\leq 1$, i.e. very mild stages of dementia. All ADRC participants are informed of the opportunity to participate in the brain bank, and as of 2010, this research clinic referral-based brain bank consisted of 607 brains from autopsies performed over 21 years. 12 patients had FTLD-tau, 13 patients had FTLD-U, and 89 patients were pathologically diagnosed as definite AD. FTLD-tau patients included 5 with Pick disease, 6 with progressive supranuclear palsy and 1 with corticobasal degeneration according to the nosologic criteria for FTLD [13, 14]. The ADRC research protocols were approved by the institutional review boards of the New York State Psychiatric Institute and of Columbia University.

\section{Clinical Assessment}

At the initial visit to our center, all cases were evaluated with medical history, physical and neurological examination, and neuropsychological test battery. A modified form of the Unified Parkinson Disease Rating Scale (mUPDRS) [15] was used to rate extrapyramidal motor signs. Patients with at least 1 motor sign rated mild-to-moderate were considered to have extrapyramidal tract signs. Visual hallucinations, depression and other behavioral symptoms were assessed using the Columbia University Scale for Psychopathology in Alzheimer's Disease [16]. CDR [12] was evaluated to rate the overall severity of dementia.

\section{Neuropsychological Assessment}

The neuropsychological test battery performed at the ADRC included the Mini-Mental State Examination (MMSE) [17], the Selective Reminding Test (SRT) [18], the Boston Naming Test, short 15-item version (BNT-15) [19], verbal fluency tests of initial letter and category [20], the Rosen Drawing Test, 5-item version (RDT-5) [21] and the Digit Span subtest from the Wechsler Memory Scale Revised [22]. Orientation was assessed by 10 items about time and place from the MMSE. SRT sub-measures included short-term recall (SRT-STR), long-term retrieval (SRT-LTR), delayed free recall (SRT-DR) and delayed recognition (SRTDRcg). An SRT retention score (=delayed recall number/correct retrieval number at last trial of encoding phase) was also calculated. The Controlled Oral Word Association test included the letter verbal fluency test using C, F and L for English speakers and A, B and S for Spanish speakers (7 AD cases) [20], and the semantic category fluency task used animal naming. The $t$ scores of all neuropsychological tests were applied based on established age- and education-adjusted norms [23, 24]. 
Yoshizawa et al.: Presenting Neuropsychological Testing Profile of Autopsy-Confirmed Frontotemporal Lobar Degeneration

\section{Neuropathological Evaluation}

Neuropathological assessment was performed using a protocol described by Vonsattel et al. [25, 26]. Neuritic plaques and neurofibrillary tangles were detected using hematoxylin-eosin and the modified Bielschowsky silver method. Immunohistochemistry was performed for beta amyloid, phosphorylated tau, ubiquitin (p62), TDP-43 and glial fibrillary acidic protein. Autopsy-confirmed AD patients have been previously described [27]. Briefly, all of the AD cases met the criteria of Braak stage $\geq 4$ neurofibrillary pathological findings and also met the CERAD neuropathological criteria for definite AD. The neuropathological diagnosis of FTLD-tau was based on the presence of tau-positive inclusions: Pick body (Pick disease), tau-positive tufted astrocytes (progressive supranuclear palsy) and ballooned achromatic neurons and astrocytic plaques (corticobasal degeneration). The neuropathological diagnosis of FTLD-U was based on the presence of typical TDP-43-type cytoplasmic ubiquitinated inclusions. Cases with FTLD did not demonstrate significant AD pathology (Braak stage $\leq 3$ and NIA-Reagan criteria low likelihood).

\section{Statistical Analyses}

Baseline characteristics were compared among the groups using Pearson's $\chi^{2}$ test or Fisher's exact test for nominal data. For continuous variables, Mann-Whitney test and Kruskal-Wallis test were used to assess differences between AD and FTLD (FTLD-tau + FTLD-U), and differences among AD, FTLD-tau and FTLD-U, respectively. To identify the underlying factor structure, a factor analysis was performed on 11 neuropsychological variables using the generalized least squares method with Promax rotation. Factor extraction was based on the Kaiser-Guttman rule of retaining components with eigenvalues $>1$. Factor loading of each test $>0.35$ was considered as a significant contributor to the factor. Mann-Whitney test and Kruskal-Wallis test were applied to each factor score and the age- and education-adjusted t values of each neuropsychological test to examine the differences between AD and FTLD, and the differences among AD, FTLD-tau and FTLD-U, respectively.

To assess the relative impairments in letter fluency compared to category fluency in FTLD, paired t tests after repeated measures analysis of variance were applied under the test condition (Letter Fluency or Category Fluency). An unpaired t test was used to compare the result of each fluency test between AD and FTLD. The 'semantic index score' [semantic index = Category Fluency/(Category Fluency + Letter Fluency)] was calculated as suggested by Rascovsky et al. [28].

The contribution of each neuropsychological variable to the distinction between autopsy-confirmed AD and FTLD was examined by selecting age- and education-adjusted t values of 11 neuropsychological variables with $\mathrm{p}<0.05$ in the univariate analysis and included them simultaneously in a multiple logistic regression analysis using a forward selection method. To identify independent predictors for differentiation of FTLD-U from AD, for differentiation of FTLD-tau from AD and for differentiation of FTLD-U from FTLD-tau, separate logistic regression analyses were performed. Analyses were conducted using SPSS version 19 (IBM Corporation, New York, N.Y., USA) and statistical significance was defined as $\mathrm{p}=0.05$.

\section{Results}

\section{Comparison of Clinical Features}

Demographic data is shown in table 1. The FTLD group was younger than the AD group at onset, initial visit and death. Subgroup analysis revealed that both the FTLD-tau and the FTLD-U group showed younger age of onset compared with the AD group. Time between onset of symptoms and death was shorter in patients with FTLD-U compared to patients with AD. The FTLD group showed an increased frequency of extrapyramidal tract signs at initial presentation compared with the AD group (Fisher's exact test; $p<0.001$; table 1). Subgroup analysis showed a higher rate of extrapyramidal tract signs in both the FTLD-tau and the FTLD-U group compared with the AD group (Fisher's exact test; $p=0.001$, post hoc analysis; $p=0.011$ and $p=0.001$, respectively). There were no group differences with regard to other symptoms, including depression.

The retrospective review of clinical data of all FTLD patients showed that all of the Pick patients presented with behavioral or personality changes, whereas patients with corticobasal degeneration, progressive supranuclear palsy and FTLD-U presented with heterogeneous cognitive symptoms such as language dysfunction, memory disturbance 
Table 1. Demographics of the patient groups

\begin{tabular}{|c|c|c|c|c|c|c|c|c|c|c|}
\hline & \multicolumn{8}{|c|}{ Pathological group } & \multirow{2}{*}{\multicolumn{2}{|c|}{$\mathrm{p}$ value }} \\
\hline & \multirow[b]{2}{*}{$\begin{array}{l}\mathrm{AD} \\
(\mathrm{n}=89)\end{array}$} & \multirow[b]{2}{*}{$\mathrm{n}$} & \multirow[b]{2}{*}{$\begin{array}{l}\text { FTLD } \\
(\mathrm{n}=25)\end{array}$} & \multirow[b]{2}{*}{$\mathrm{n}$} & \multicolumn{4}{|c|}{ FTLD subtype } & & \\
\hline & & & & & $\begin{array}{l}\text { FTLD-tau } \\
(\mathrm{n}=12)\end{array}$ & $\mathrm{n}$ & $\begin{array}{l}\text { FTLD-U } \\
(\mathrm{n}=13)\end{array}$ & $\mathrm{n}$ & AD/FTLD & $\begin{array}{l}\text { AD/FTLD-tau/ } \\
\text { FTLD-U }\end{array}$ \\
\hline \multicolumn{11}{|l|}{ Demographic data } \\
\hline Age of onset & \multicolumn{2}{|l|}{$68.3 \pm 9.8$} & \multicolumn{2}{|l|}{$58.1 \pm 9.4$} & \multicolumn{2}{|l|}{$60.0 \pm 10.4$} & \multicolumn{2}{|l|}{$56.5 \pm 8.7$} & $<0.001^{\ddagger}, \mathrm{a}$ & $<0.001^{\S, b, c}$ \\
\hline Age at initial visit & \multicolumn{2}{|l|}{$71.6 \pm 9.6$} & \multicolumn{2}{|c|}{$63.6 \pm 10.5$} & \multicolumn{2}{|l|}{$67.2 \pm 11.5$} & \multicolumn{2}{|l|}{$60.2 \pm 8.6$} & $0.001^{\text {t, a }}$ & $0.001^{\S, c}$ \\
\hline Age at death & \multicolumn{2}{|l|}{$78.6 \pm 10.4$} & \multicolumn{2}{|c|}{$68.8 \pm 11.2$} & \multicolumn{2}{|l|}{$74.0 \pm 10.2$} & \multicolumn{2}{|l|}{$63.9 \pm 10.2$} & $<0.001^{\ddagger}, \mathrm{a}$ & $<0.001^{\S, c, d}$ \\
\hline Disease duration, years & \multicolumn{2}{|l|}{$10.3 \pm 4.1$} & \multicolumn{2}{|c|}{$9.8 \pm 7.4$} & \multicolumn{2}{|l|}{$12.6 \pm 7.5$} & \multicolumn{2}{|l|}{$7.4 \pm 6.7$} & $0.10^{\ddagger}$ (n.s.) & $0.018^{\S, c}$ \\
\hline Education, years & \multicolumn{2}{|l|}{$14.7 \pm 4.3$} & \multicolumn{2}{|l|}{$15.7 \pm 2.9$} & \multicolumn{2}{|l|}{$14.3 \pm 2.6$} & \multicolumn{2}{|l|}{$17.0 \pm 2.7$} & $0.55^{\ddagger}$ (n.s.) & $0.071^{\S}$ (n.s.) \\
\hline Male gender, $\%$ & \multicolumn{2}{|l|}{47.2} & \multicolumn{2}{|l|}{68.0} & \multicolumn{2}{|l|}{66.7} & \multicolumn{2}{|l|}{69.2} & $0.066^{*}$ (n.s.) & $0.18^{\dagger}$ (n.s.) \\
\hline $\begin{array}{l}\text { Race (White/Black/Hispanic/ } \\
\text { other) }\end{array}$ & \multicolumn{2}{|l|}{$73 / 6 / 9 / 1$} & $24 / 1 / 0 / C$ & & $12 / 0 / 0 / 0$ & & $12 / 1 / 0 / c$ & & $0.40^{\dagger}$ (n.s.) & $0.78^{\dagger}$ (n.s.) \\
\hline Blessed Functional Activity Scale & $2.8 \pm 1.7$ & & $3.0 \pm 2.6$ & & $4.1 \pm 2.9$ & & $2.0 \pm 1.8$ & & $0.69^{\ddagger}$ (n.s.) & $0.099^{\S}$ (n.s.) \\
\hline MMSE & $21.2 \pm 4.8$ & & $23.7 \pm 4.0$ & & $23.0 \pm 4.7$ & & $24.3 \pm 3.3$ & & $0.021^{\ddagger}, \mathrm{a}$ & $0.060^{\S}$ (n.s.) \\
\hline CDR & $0.74 \pm 0.2 \varepsilon$ & & $0.64 \pm 0.3$ & & $0.58 \pm 0.36$ & & $0.69 \pm 0.3$ & & $0.16^{\ddagger}$ (n.s.) & $0.27^{\S}$ (n.s.) \\
\hline Clinical symptoms & & & & & & & & & & \\
\hline Extrapyramidal sign & $9.9 \%$ & 81 & $50.0 \%$ & 20 & $41.7 \%$ & 12 & $62.5 \%$ & 8 & $<0.001^{\dagger, \mathrm{a}}$ & $<0.001^{\dagger, b, c}$ \\
\hline Visual hallucination & $4.2 \%$ & 71 & $0 \%$ & 19 & $0 \%$ & 9 & $0 \%$ & 10 & $1.00^{\dagger}$ (n.s.) & $1.00^{\dagger}$ (n.s.) \\
\hline Illusion & $4.3 \%$ & 70 & $0 \%$ & 19 & $0 \%$ & 9 & $0 \%$ & 10 & $1.00^{\dagger}$ (n.s.) & $1.00^{\dagger}$ (n.s.) \\
\hline Delusion & $7.7 \%$ & 56 & $5.3 \%$ & 19 & $11.1 \%$ & 9 & $0 \%$ & 10 & $1.00^{\dagger}$ (n.s.) & $0.57^{\dagger}$ (n.s.) \\
\hline Agitation & $9.8 \%$ & 51 & $11.1 \%$ & 18 & $12.5 \%$ & 8 & $10.0 \%$ & 10 & $1.00^{\dagger}$ (n.s.) & $1.00^{\dagger}$ (n.s.) \\
\hline Depression & $7.8 \%$ & 51 & $11.1 \%$ & 18 & $12.5 \%$ & 8 & $10.0 \%$ & 10 & $0.65^{\dagger}$ (n.s.) & $0.80^{\dagger}$ (n.s.) \\
\hline
\end{tabular}

Continuous variables are presented as means \pm SD. Categorical variables are reported as percentage or number of patients.

* Pearson $\chi^{2} .{ }^{\dagger}$ Fisher's exact test. ${ }^{\ddagger} \mathrm{U}$ of Mann-Whitney. ${ }^{\S} \chi^{2}$ of Kruskal-Wallis.

${ }^{\mathrm{a}}$ AD vs. FTLD. ${ }^{\mathrm{b}}$ AD vs. FTLD-tau. ${ }^{\mathrm{c}}$ AD vs. FTLD-U. ${ }^{\mathrm{d}}$ FTLD-tau vs. FTLD-U.

and depression, as well as motor symptoms in some cases (online suppl. table 1, see www. karger.com/doi/10.1159/000353860). Abnormalities in expressive language function were present in 5/11 (45.5\%) FTLD-tau and in 6/13 (46.2\%) FTLD-U patients. No patients presented with clinical features of semantic dementia. Motor neuron disease findings were ultimately present in $12 / 13$ (92.3\%) FTLD-U patients, but only 3 patients initially presented with such findings.

\section{Neuropsychological Testing Domains Using Factor Analysis}

The explanatory factor analysis solutions with the $t$ values of all 11 neuropsychological tests revealed a factor structure consisting of three distinctive factors. The loadings from the rotated solution are shown in table 2 (upper part; rotated eigenvalues $=2.69,2.35$ and 1.89, respectively). The three factors were representative of memory, language and attention domain, according to the neuropsychological subscores from which high factor loadings were extracted. The correlation between memory factor and language factor was 0.300 and the correlation between language and attention factor was 0.436 . However, the correlation between memory factor and attention factor was not significant (table 2, lower part).

Table 3 (upper part) shows that the memory factor score in the AD group was lower than that in the FTLD group $(\mathrm{p}=0.024)$, whereas the language factor and attention factor scores in the FTLD group were lower than those in the AD group $(p=0.002$ and $p=0.015$, respectively). Subgroup analysis demonstrated that language and attention factor scores in the FTLD-U group were significantly lower than in the AD group $(p=0.006$ and $p=0.005$, respectively). 
Dementia

Cognitive Disorders

Table 2. Factor analysis of neuropsychological tests

\begin{tabular}{l|l}
\hline Dement Geriatr Cogn Disord 2013;36:279-289 \\
\hline DOI: $\underline{10.1159 / 000353860}$ & $\begin{array}{l}\text { @ 2013 S. Karger AG, Basel } \\
\text { www.karger.com/dem }\end{array}$ \\
\hline
\end{tabular}

Yoshizawa et al.: Presenting Neuropsychological Testing Profile of Autopsy-Confirmed Frontotemporal Lobar Degeneration

\begin{tabular}{lccc}
\hline & $\begin{array}{c}\text { Factor } 1: \\
\text { memory }\end{array}$ & $\begin{array}{r}\text { Factor 2: } \\
\text { language }\end{array}$ & $\begin{array}{r}\text { Factor 3: } \\
\text { attention }\end{array}$ \\
\hline SRT-DR & $\mathbf{0 . 9 1 1}$ & -0.133 & 0.014 \\
SRT-LTR & $\mathbf{0 . 8 6 7}$ & 0.057 & -0.031 \\
SRT-DRcg & $\mathbf{0 . 7 0 2}$ & 0.022 & 0.153 \\
Orientation & $\mathbf{0 . 4 1 2}$ & 0.293 & -0.070 \\
Category Fluency & 0.182 & $\mathbf{0 . 8 1 4}$ & -0.030 \\
Letter Fluency & -0.010 & $\mathbf{0 . 6 6 7}$ & 0.185 \\
RDT-5 & -0.058 & $\mathbf{0 . 4 2 9}$ & -0.104 \\
BNT-15 & 0.245 & $\mathbf{0 . 3 6 5}$ & -0.126 \\
SRT-STR & -0.290 & $\mathbf{0 . 3 5 6}$ & $\mathbf{0 . 3 6 4}$ \\
Digit Span Forward & 0.078 & -0.250 & $\mathbf{0 . 8 8 2}$ \\
Digit Span Backward & 0.072 & 0.118 & $\mathbf{0 . 7 5 5}$ \\
\hline Eigenvalue & 2.69 & 2.35 & 1.89 \\
\hline & Factor 1 & Factor 2 & Factor 3 \\
\hline Factor 1 & 1.000 & 0.300 & 0.011 \\
Factor 2 & & 1.000 & 0.436 \\
Factor 3 & & & 1.000 \\
\hline
\end{tabular}

Factor loadings $\geq 0.35$ are in bold.

Table 3. Results of neuropsychological tests

\begin{tabular}{|c|c|c|c|c|c|c|}
\hline & \multicolumn{4}{|c|}{ Pathological group } & & \\
\hline & \multirow[b]{2}{*}{$\begin{array}{l}\mathrm{AD} \\
(\mathrm{n}=89)\end{array}$} & \multirow[b]{2}{*}{$\begin{array}{l}\text { FTLD } \\
(n=25)\end{array}$} & \multicolumn{2}{|l|}{ FTLD subtype } & \multicolumn{2}{|l|}{$\mathrm{p}$ value } \\
\hline & & & $\begin{array}{l}\text { FTLD-tau } \\
(\mathrm{n}=12)\end{array}$ & $\begin{array}{l}\text { FTLD-U } \\
(n=13)\end{array}$ & AD/FTLD & $\begin{array}{l}\text { AD/ } \\
\text { FTLD-tau/ } \\
\text { FTLD-U }\end{array}$ \\
\hline Memory factor & $-0.13 \pm 0.80$ & $0.46 \pm 1.27$ & $0.39 \pm 1.39$ & $0.52 \pm 1.21$ & $0.024^{\mathrm{a}}$ & 0.071 \\
\hline Language factor & $0.14 \pm 0.92$ & $-0.49 \pm 0.71$ & $-0.44 \pm 0.79$ & $-0.53 \pm 0.67$ & $0.002^{\mathrm{a}}$ & $0.006^{\mathrm{c}}$ \\
\hline Attention factor & $0.088 \pm 0.93$ & $-0.31 \pm 0.72$ & $-0.097 \pm 0.86$ & $-0.51 \pm 0.52$ & $0.015^{\mathrm{a}}$ & $0.016^{\mathrm{c}}$ \\
\hline \multicolumn{7}{|l|}{ Attention } \\
\hline Digit Span Forward & $5.7 \pm 1.2$ & $5.7 \pm 0.68$ & $5.7 \pm 0.78$ & $5.8 \pm 0.60$ & 0.63 & 0.72 \\
\hline Digit Span Backward & $3.5 \pm 1.2$ & $3.4 \pm 1.1$ & $3.4 \pm 1.4$ & $3.3 \pm 0.63$ & $0.031^{\mathrm{a}}$ & $0.027^{\mathrm{c}}$ \\
\hline SRT-STR & $15.8 \pm 5.9$ & $12.1 \pm 6.2$ & $14.8 \pm 6.3$ & $9.6 \pm 5.2$ & 0.067 & $0.021^{\mathrm{c}}$ \\
\hline \multicolumn{7}{|l|}{ Memory } \\
\hline SRT-LTR & $8.4 \pm 8.2$ & $15.5 \pm 14.6$ & $13.3 \pm 14.7$ & $17.5 \pm 14.9$ & 0.38 & 0.60 \\
\hline SRT-DR & $1.2 \pm 1.9$ & $4.1 \pm 3.4$ & $3.8 \pm 3.5$ & $4.3 \pm 3.4$ & $0.002^{\mathrm{a}}$ & $0.008^{\mathrm{c}}$ \\
\hline SRT-DRcg & $7.6 \pm 3.0$ & $9.3 \pm 2.8$ & $9.3 \pm 3.2$ & $9.3 \pm 2.5$ & 0.098 & 0.24 \\
\hline \multicolumn{7}{|l|}{ Language } \\
\hline Category Fluency & $10.2 \pm 6.2$ & $8.9 \pm 5.1$ & $8.4 \pm 4.9$ & $9.3 \pm 5.5$ & 0.12 & 0.30 \\
\hline Letter Fluency & $10.5 \pm 5.6$ & $5.7 \pm 4.1$ & $6.2 \pm 4.5$ & $5.2 \pm 3.7$ & $<0.001^{\mathrm{a}}$ & $<0.001^{\mathrm{b}, \mathrm{c}}$ \\
\hline Orientation & $6.9 \pm 2.5$ & $8.4 \pm 1.9$ & $7.8 \pm 2.0$ & $8.9 \pm 1.8$ & $0.013^{\mathrm{a}}$ & $0.018^{c}$ \\
\hline BNT-15 & $12.8 \pm 2.0$ & $12.2 \pm 4.6$ & $12.3 \pm 4.5$ & $12.0 \pm 4.8$ & 0.72 & 0.68 \\
\hline \multicolumn{7}{|l|}{ Visuospatial } \\
\hline RDT-5 & $3.1 \pm 1.1$ & $2.7 \pm 1.4$ & $2.1 \pm 1.4$ & $3.2 \pm 1.2$ & $0.036^{\mathrm{a}}$ & $0.033^{\mathrm{b}}$ \\
\hline
\end{tabular}

Continuous variables are reported as means \pm SD. Statistics was performed using age- and educationadjusted t score. ${ }^{\mathrm{a}}$ AD vs. FTLD. ${ }^{\mathrm{b}}$ AD vs. FTLD-tau. ${ }^{\mathrm{c}}$ AD vs. FTLD-U. 


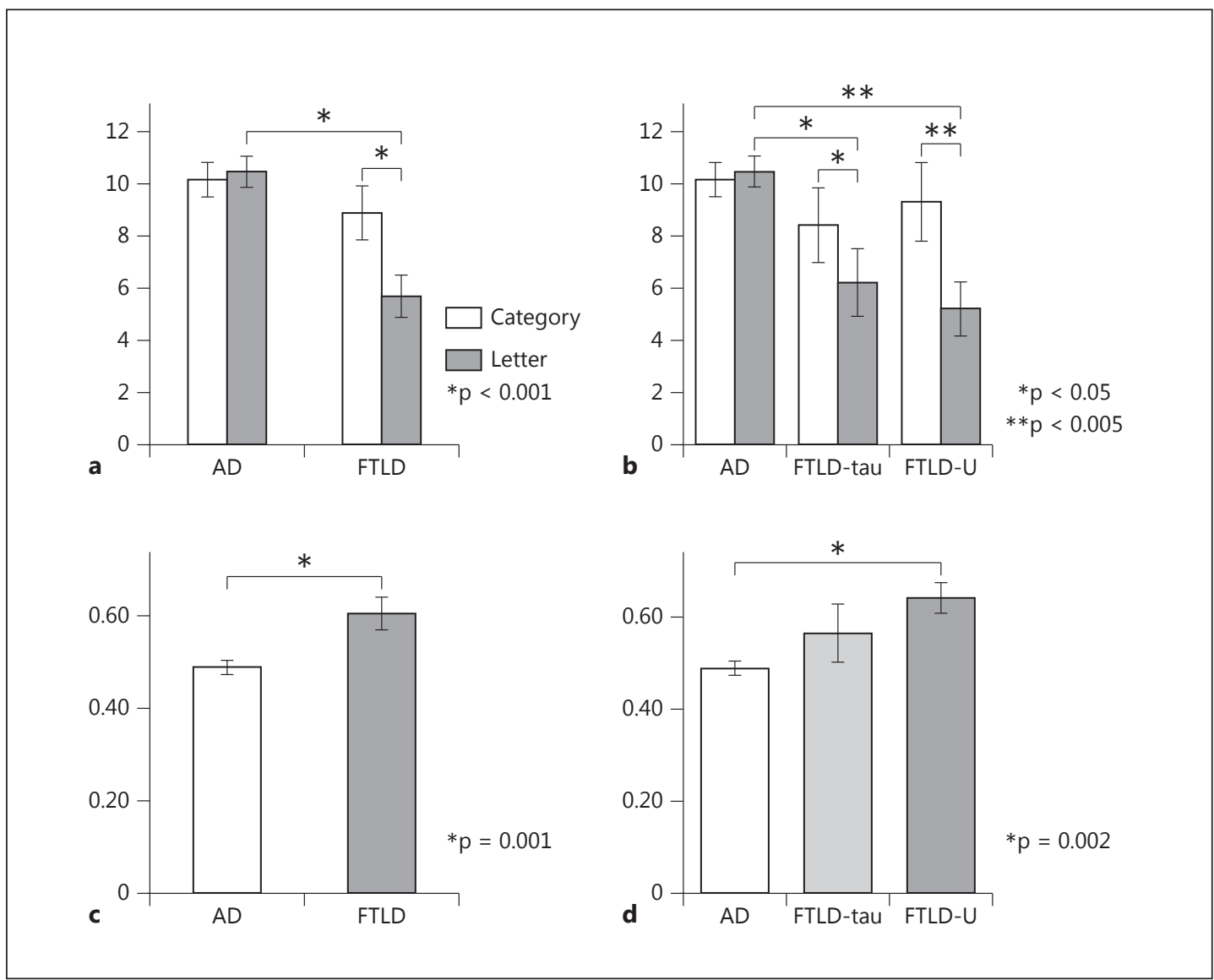

Fig. 1. FTLD scores compared with AD. a, b Letter and Category Fluency scores. c, d Semantic index [Category Fluency/(Category Fluency + Letter Fluency)] scores. Brackets show the significant differences between scores. Error bars represent standard error of the mean.

\section{Comparison of Neuropsychological Testing}

As shown in table 3 (lower part), the FTLD group showed, in comparison to the AD group, significantly lower scores on Digit Span Backward $(p=0.031)$, Letter Fluency $(p<0.001)$ and RDT-5 ( $p=0.036)$ tests, but higher scores on SRT-DR $(p=0.002)$ and Orientation $(p=0.013)$. In the subgroup analysis, the FTLD-U group showed higher scores on SRT-DR $(p=0.011)$ and Orientation ( $p=0.005)$, and lower scores on Digit Span Backward ( $\mathrm{p}=0.007)$, SRT-STR ( $\mathrm{p}=$ $0.006)$ and Letter Fluency $(\mathrm{p}<0.001)$ compared with AD patients. The FTLD-tau group's scores on RDT-5 and Letter Fluency were lower than those of the AD group ( $p=0.010$ and $\mathrm{p}=0.006$, respectively).

The memory retention score (=delayed recall number/correct retrieval number at last trial of encoding phase) was lower in the $\mathrm{AD}$ patients (mean $=0.20, \mathrm{SD}=0.29$ ) than in the FTLD patients (mean $=0.60, \mathrm{SD}=0.48$ ); Mann-Whitney $\mathrm{U}=524.5, \mathrm{p}<0.001$. Kruskal-Wallis test and post hoc separate analyses of FTLD-tau and FTLD-U revealed that retention scores in $\mathrm{AD}$ patients (mean $=0.20, \mathrm{SD}=0.29$ ) were lower for both FTLD-tau (mean $=0.63, \mathrm{SD}=0.52$, $\mathrm{p}=0.003)$ and FTLD-U patients (mean $=0.57, \mathrm{SD}=0.44, \mathrm{p}=0.005)$. 
Yoshizawa et al.: Presenting Neuropsychological Testing Profile of Autopsy-Confirmed Frontotemporal Lobar Degeneration

Table 4. Multivariate logistic model comparisons

\begin{tabular}{|c|c|c|c|c|c|}
\hline & \multirow{2}{*}{$\begin{array}{l}\text { Partial regres- } \\
\text { sion coefficient }\end{array}$} & \multirow{2}{*}{$\begin{array}{l}\mathrm{p} \\
\text { value }\end{array}$} & \multirow[t]{2}{*}{$\mathrm{OR}$} & \multicolumn{2}{|c|}{$95 \% \mathrm{CI}$} \\
\hline & & & & lower & upper \\
\hline \multicolumn{6}{|l|}{ FTLD-U vs. AD } \\
\hline SRT-DR & 0.145 & 0.001 & 1.156 & 1.062 & 1.257 \\
\hline Letter Fluency & -0.171 & 0.003 & 0.843 & 0.752 & 0.944 \\
\hline Constant & 0.100 & 0.964 & & & \\
\hline \multicolumn{6}{|l|}{ FTLD-tau vs. AD } \\
\hline RDT-5 & -0.085 & 0.019 & 0.919 & 0.856 & 0.986 \\
\hline SRT-STR & 0.210 & 0.049 & 1.233 & 1.001 & 1.520 \\
\hline SRT-DR & 0.159 & 0.001 & 1.173 & 1.067 & 1.289 \\
\hline Letter Fluency & -0.123 & 0.004 & 0.884 & 0.812 & 0.962 \\
\hline Constant & -9.386 & 0.095 & & & \\
\hline \multicolumn{6}{|c|}{ FTLD-U vs. FTLD-tau } \\
\hline RDT-5 & 0.095 & 0.063 & 1.099 & 0.995 & 1.215 \\
\hline SRT-STR & -0.256 & 0.054 & 0.774 & 0.597 & 1.004 \\
\hline Constant & 8.637 & 0.146 & & & \\
\hline
\end{tabular}

\section{Verbal Fluency Tests}

Letter and Category Fluency task performance differed in FTLD and AD patients, with repeated measures analysis of covariance for fluency test revealing a significant two-way interaction of diagnosis by fluency $[F(1,112)=9.27, p=0.003]$. In Letter Fluency, FTLD was impaired compared to AD [t $(112)=3.93, \mathrm{p}<0.001]$, whereas in Category Fluency, there was no significant difference between FTLD and AD [t (112) $=0.942, p=0.35]$ (fig. 1a). As shown in figure $1 \mathrm{~b}$, in subgroup analysis, Letter Fluency in the FTLD-tau and FTLD-U groups was impaired more than that in the $\operatorname{AD}$ group $[F(2,111)=7.78, p=0.001$, post hoc test; $p=$ 0.035 and $\mathrm{p}=0.004$, respectively]. The semantic index was significantly lower in AD patients ( mean $=0.49, \mathrm{SD}=0.14)$ compared with FTLD patients (mean $=0.60, \mathrm{SD}=0.18)[\mathrm{t}(112)=$ $3.41, p=0.001$ ] (fig. 1c). Subgroup analysis showed that the semantic index in AD patients (mean $=0.49, \mathrm{SD}=0.14$ ) was significantly lower than that in FTLD-U patients (mean $=0.64$, $S D=0.12)(p=0.002)$ (fig. 1d).

\section{Predictive Value of Neuropsychological Tests}

Logistic regression analysis using a stepwise forward selection method showed that the SRT-DR $(\mathrm{p}<0.001, \mathrm{OR}=1.179,95 \% \mathrm{CI}=1.095-1.269)$, the BNT-15 $(\mathrm{p}=0.020, \mathrm{OR}=0.966$, $95 \% \mathrm{CI}=0.939-0.995)$ and Letter Fluency $(\mathrm{p}<0.001, \mathrm{OR}=0.881,95 \% \mathrm{CI}=0.823-0.943)$ remained as independent predictors of FTLD compared to AD in the final model. With this model, the diagnostic sensitivity for FTLD was $64.0 \%$, while specificity was $95.5 \%$ and accuracy $88.6 \%$. Separate logistic regression analyses showed that SRT-DR and Letter Fluency remained as independent predictors of FTLD-U compared to AD (sensitivity 58.3\%, specificity 98.9\%, accuracy 94.1\%), and also showed that RDT-5, SRT-STR, SRT-DR and Letter Fluency remained as independent predictors of FTLD-tau compared to AD (sensitivity 46.2\%, specificity 98.9\%, accuracy 92.2\%). Although RDT-5 and SRT-STR remained as independent predictors of FTLD-U compared to FTLD-tau in the final model, they did not reach statistical significance $(p=0.063$ and $p=0.054$, respectively; table 4). 
Dementia

and Geriatric

Cognitive Disorders

\begin{tabular}{l|l}
\hline \multicolumn{2}{l|}{ Dement Geriatr Cogn Disord 2013;36:279-289 } \\
\hline DOI: $\underline{10.1159 / 000353860}$ & $\begin{array}{l}\text { @ } 2013 \text { S. Karger AG, Basel } \\
\text { www.karger.com/dem }\end{array}$ \\
\hline
\end{tabular}

Yoshizawa et al.: Presenting Neuropsychological Testing Profile of Autopsy-Confirmed Frontotemporal Lobar Degeneration

\section{Discussion}

We compared neuropsychological features at initial presentation in patients with early stages of dementia (CDR $\leq 1$ ) eventually confirmed by autopsy as having suffered from FTLD or AD. Some prior studies have indicated that the neuropsychological characteristics of FTLD include early changes in verbal fluency, planning, working memory and executive function, in comparison to the more significant deficits in episodic memory, visuospatial function and praxes characteristic of AD [6,29-31]. However, other studies have failed to find such differences between AD and FTLD [5, 32-34]. These discrepancies may relate to different neuropsychological measures used, small sample sizes, difficulties with clinical classification and heterogeneity of FTLD. To elucidate the validity of neuropsychological testing in differentiating FTLD from $\mathrm{AD}$, we restricted our analysis to subjects with early stages of disease (CDR $\leq 1$ ) and examined the results using factor analysis of neuropsychological test scores, as well as examining components of memory testing.

Factor analysis showed three latent factors: memory, language and attention. Comparison of the factor scores showed dissociative patterns, with the FTLD patients performing better on the memory factor and worse on the language and attention factors compared with the AD patients. Factor analysis allows us to reduce a large amount of complicated data of neuropsychological scores, to evaluate the construct validity of a test battery adopted, and to reveal the cognitive background of the diseases $[35,36]$. Therefore, data reduction using factor analysis and investigating affected cognitive domains would be useful for discriminating between diseases whose neuropsychological manifestations mimic each other.

Memory test sub-measures were useful in discriminating FTLD from AD. FTLD patients showed preserved memory retention compared with AD patients, although the other submeasures of SRT such as long-term retrieval and delayed recognition in FTLD patients were comparable to those in AD patients. Examining FTLD-U and FTLD-tau separately, short-term recall only in FTLD-U was significantly lower than in AD. Considering three aspects of episodic long-term memory, including encoding, storage and retrieval, SRT sub-measures allow assessment of working memory by short-term recall, memory encoding by long-term retrieval, and memory retrieval by delayed recall [18]. Our results suggest that FTLD patients show preserved abilities in the memory storage and retrieval phases compared with AD patients, whereas FTLD patients, especially FTLD-U patients, show more deficits in working memory. Prior studies have been inconsistent in this regard, with some reporting that the memory disturbance of FTLD is similar to that of AD [37], while others have reported differences between FTLD and AD for encoding and forgetting rate in word list memory tests [38]. Memory disturbance in FTLD may result from frontal-executive impairments such as inefficient memory strategies and working memory rather than storage or recall deficits themselves [39] or impaired access to semantic representations [40]. This contrasts with memory impairment in $\mathrm{AD}$, which likely relates principally to encoding impairment due to entorhinal cortex and hippocampus dysfunction present at the earliest stages of the disease [41]. These pathological differences are likely responsible for the difference in memory sub-measures between the groups, which may allow better differentiation of FTLD from AD.

Verbal fluency tests also allowed some distinction between FTLD and AD. Our results showed that both types of verbal fluency in FTLD were more affected than in AD. However, Letter Fluency was more impaired than Category Fluency in FTLD compared with AD. Prior studies have also shown that Letter Fluency may be more impaired than Category Fluency in FTLD compared with AD [42, 43]. Rascovsky et al. [28] have shown disparate phonemic letter fluency and semantic category fluency deficits in autopsy-confirmed FTLD. Semantic fluency likely depends on the integrity of semantic memory, which demands the temporal-lobemediated semantic system, whereas phonemic fluency may be more sensitive to executive 
Yoshizawa et al.: Presenting Neuropsychological Testing Profile of Autopsy-Confirmed Frontotemporal Lobar Degeneration

dysfunction, which may be caused by frontal lobe damage [44]. Thus, the differences in performance on these tasks are consistent with differing principal pathological involvement between AD with early temporal involvement and FTLD with early frontal involvement.

Discriminant analysis for FTLD versus AD using logistic regression showed that Letter Fluency, the BNT-15 and the SRT-DR were independent predictors of FTLD pathology. Although cut-off points in our study did not allow sufficient discrimination of the two groups, it is possible that combinations of certain tests measuring specific cognitive domains, such as language function and memory, might be useful to discriminate FTLD from AD. Similarly, while we were able to discriminate between FTLD-U and AD as well as between FTLD-tau and $A D$, we were unable to successfully discriminate between FTLD-U and FTLD-tau using logistic regression analysis. This may be related to the tests used or to an overlap between the impairments in these subgroups. Prior work has included some reporting differences between FTLD-tau and FTLD-U/TDP [8, 10], some failing to find such neuropsychological differences [11]. It may be necessary to incorporate measures of behavioral change, neuropsychiatric symptoms, concomitant neurological signs and neuroimaging data to make such distinctions between different FTLD pathologies.

There are several limitations to the present study. One is the selection of the neuropsychological battery. A more detailed evaluation of specific areas of function may better characterize the differences between patients with AD and FTLD. Frontal lobe functions such as executive function, judgment and personality have been particularly implicated in FTLD [6], and our battery had only limited tests with respect to these functions. Secondly, this sample was not population-based but clinic-based, and the sample size of the FTLD group was small, therefore the results may not be generalizable. Thirdly, not every case had TDP-43 staining, so heterogeneity of the FTLD-U cases is possible. Finally, we do not have sufficient longitudinal data to determine whether rate of change measures might be more useful [31].

\section{Conclusions}

Our study suggests the following: (1) FTLD patients at early symptomatic stages show lower test scores in attentional and language domains and higher test scores in memory domain than do AD patients. (2) FTLD patients show a pattern of memory subtest findings suggesting working memory deficits, whereas AD patients show delayed recall deficits. (3) FTLD patients show more impaired Letter Fluency than Category Fluency, unlike AD patients. (4) FTLD can possibly be discriminated from AD using combined test scores of Letter Fluency, the Boston Naming Test and delayed recall. (5) FTLD-U and FTLD-tau patients are not distinguishable by their neuropsychological test performances.

\section{Acknowledgements}

This study was supported by NIH grants P50AG008702 (PI M. Shelanski) and UL1RR024156 (PI H. Ginsberg), the Alzheimer's Association, the Alzheimer's Drug Discovery Foundation, the Henry P. Panasci Fund and the Taub Institute for Research on Alzheimer's Disease and the Aging Brain.

\section{Disclosure Statement}

There is no conflict of interest. 
Yoshizawa et al.: Presenting Neuropsychological Testing Profile of Autopsy-Confirmed Frontotemporal Lobar Degeneration

\section{References}

1 Whitwell JL, Jack CR Jr, Przybelski SA, Parisi JE, Senjem ML, Boeve BF, Knopman DS, Petersen RC, Dickson DW, Josephs KA: Temporoparietal atrophy: a marker of AD pathology independent of clinical diagnosis. Neurobiol Aging 2011;32:1531-1541.

2 Rabinovici G, Furst A, O'Neil J, Racine C, Mormino E, Baker S, Chetty S, Patel P, Pagliaro T, Klunk W, Mathis C, Rosen H, Miller B, Jagust W: 11C-PIB PET imaging in Alzheimer disease and frontotemporal lobar degeneration. Neurology 2007;68:1205-1212.

- 3 Bian H, Van Swieten JC, Leight S, Massimo L, Wood E, Forman M, Moore P, de Koning I, Clark CM, Rosso S, Trojanowski J, Lee VM, Grossman M: CSF biomarkers in frontotemporal lobar degeneration with known pathology. Neurology 2008;70:1827-1835.

4 Mendez MF, Shapira JS, McMurtray A, Licht E, Miller BL: Accuracy of the clinical evaluation for frontotemporal dementia. Arch Neurol 2007;64:830-835.

- 5 Hutchinson AD, Mathias JL: Neuropsychological deficits in frontotemporal dementia and Alzheimer's disease: a meta-analytic review. J Neurol Neurosurg Psychiatry 2007;78:917-928.

6 Seelaar H, Rohrer JD, Pijnenburg YA, Fox NC, van Swieten JC: Clinical, genetic and pathological heterogeneity of frontotemporal dementia: a review. J Neurol Neurosurg Psychiatry 2011;82:476-486.

- 7 Thomas-Antérion C, Jacquin K, Laurent B: Differential mechanisms of impairment of remote memory in Alzheimer's and frontotemporal dementia. Dement Geriatr Cogn Disord 2000;11:100-106.

8 Yokota O, Tsuchiya K, Arai T, Yagishita S, Matsubara O, Mochizuki A, Tamaoka A, Kawamura M, Yoshida H, Terada S, Ishizu H, Kuroda S, Akiyama H: Clinicopathological characterization of Pick's disease versus frontotemporal lobar degeneration with ubiquitin/TDP-43-positive inclusions. Acta Neuropathol 2009;117:429444.

- 9 Josephs KA, Hodges JR, Snowden JS, Mackenzie IR, Neumann M, Mann DM, Dickson DW: Neuropathological background of phenotypical variability in frontotemporal dementia. Acta Neuropathol 2011;122:137-153.

10 Forman MS, Farmer J, Johnson JK, Clark CM, Arnold SE, Coslett HB, Chatterjee A, Hurtig HI, Karlawish JH, Rosen HJ, Van Deerlin V, Lee VM, Miller BL, Trojanowski JQ, Grossman M: Frontotemporal dementia: clinicopathological correlations. Ann Neurol 2006;59:952-962.

11 Mendez MF, Joshi A, Tassniyom K, Teng E, Shapira JS: Clinicopathologic differences among patients with behavioral variant frontotemporal dementia. Neurology 2013;80:561-568.

12 Morris JC: The Clinical Dementia Rating (CDR): current version and scoring rules. Neurology 1993;43:24122414.

13 Cairns NJ, Bigio EH, Mackenzie IR, Neumann M, Lee VM, Hatanpaa KJ, White CL, Schneider JA, Grinberg LT, Halliday G, Duyckaerts C, Lowe JS, Holm IE, Tolnay M, Okamoto K, Yokoo H, Murayama S, Woulfe J, Munoz DG, Dickson DW, Ince PG, Trojanowski JQ, Mann DM: Neuropathologic diagnostic and nosologic criteria for frontotemporal lobar degeneration: consensus of the Consortium for Frontotemporal Lobar Degeneration. Acta Neuropathol 2007;114:5-22.

14 Mackenzie IR, Neumann M, Bigio EH, Cairns NJ, Alafuzoff I, Kril J, Kovacs GG, Ghetti B, Halliday G, Holm IE, Ince PG, Kamphorst W, Revesz T, Rozemuller AJ, Kumar-Singh S, Akiyama H, Baborie A, Spina S, Dickson DW, Trojanowski JQ, Mann DM: Nomenclature for neuropathologic subtypes of frontotemporal lobar degeneration: consensus recommendations. Acta Neuropathol 2009;117:15-18.

15 Richards M, Marder K, Bell K, Dooneief G, Mayeux R, Stern Y: Interrater reliability of extrapyramidal signs in a group assessed for dementia. Arch Neurol 1991;48:1147-1149.

16 Devanand DP, Miller L, Richards M, Marder K, Bell K, Mayeux R, Stern Y: The Columbia University Scale for Psychopathology in Alzheimer's Disease. Arch Neurol 1992;49:371-376.

17 Folstein M, Folstein S, McHugh P: 'Mini-mental state'. A practical method for grading the cognitive state of patients for the clinician. J Psychiatr Res 1975;12:189-198.

-18 Buschke H, Fuld PA: Evaluating storage, retention, and retrieval in disordered memory and learning. Neurology 1974;24:1019-1025.

19 Kaplan E, Goodglass H, Weintraub S: Boston Naming Test. Philadelphia, Lea \& Febiger, 1983.

20 Benton AL, Hamsher K: Multilingual Aphasia Examination. Iowa City, University of Iowa, 1976.

21 Rosen W: The Rosen Drawing Test. Bronx, Veterans Administration Medical Center, 1981.

22 Wechsler D: Wechsler Memory Scale-Revised Manual. San Antonio, The Psychological Corporation, 1987.

-23 Stricks L, Pittman J, Jacobs DM, Sano M, Stern Y: Normative data for a brief neuropsychological battery administered to English- and Spanish-speaking community-dwelling elders. J Int Neuropsychol Soc 1998;4:311318.

24 Weintraub S, Salmon D, Mercaldo N, Ferris S, Graff-Radford NR, Chui H, Cummings J, DeCarli C, Foster NL, Galasko D, Peskind E, Dietrich W, Beekly DL, Kukull WA, Morris JC: The Alzheimer's Disease Centers' Uniform Data Set (UDS): the neuropsychologic test battery. Alzheimer Dis Assoc Disord 2009;23:91-101.

25 Vonsattel JP, Aizawa H, Ge P, DiFiglia M, McKee AC, MacDonald M, Gusella JF, Landwehrmeyer GB, Bird ED, Richardson EP: An improved approach to prepare human brains for research. J Neuropathol Exp Neurol 1995; 54:42-56

26 Vonsattel JP, Del Amaya MP, Keller CE: Twenty-first century brain banking. Processing brains for research: The Columbia University methods. Acta Neuropathol 2008;115:509-532. 
Yoshizawa et al.: Presenting Neuropsychological Testing Profile of Autopsy-Confirmed Frontotemporal Lobar Degeneration

27 Yoshizawa H, Vonsattel JP, Honig LS: Early neuropsychological discriminants for Lewy body disease: an autopsy series. J Neurol Neurosurg Psychiatry 2013, E-pub ahead of print.

28 Rascovsky K, Salmon DP, Hansen LA, Thal LJ, Galasko D: Disparate letter and semantic category fluency deficits in autopsy-confirmed frontotemporal dementia and Alzheimer's disease. Neuropsychology 2007;21:20-30.

29 Harciarek M, Jodzio K: Neuropsychological differences between frontotemporal dementia and Alzheimer's disease: a review. Neuropsychol Rev 2005;15:131-145.

30 Piguet 0, Hornberger M, Mioshi E, Hodges JR: Behavioural-variant frontotemporal dementia: diagnosis, clinical staging, and management. Lancet Neurol 2011;10:162-172.

-31 Binetti G, Locascio JJ, Corkin S, Vonsattel JP, Growdon JH: Differences between Pick disease and Alzheimer disease in clinical appearance and rate of cognitive decline. Arch Neurol 2000;57:225-232.

-32 Collette F, Amieva H, Adam S, Hogge M, Van der Linden M, Fabrigoule C, Salmon E: Comparison of inhibitory functioning in mild Alzheimer's disease and frontotemporal dementia. Cortex 2007;43:866-874.

-33 Giovagnoli AR, Erbetta A, Reati F, Bugiani O: Differential neuropsychological patterns of frontal variant frontotemporal dementia and Alzheimer's disease in a study of diagnostic concordance. Neuropsychologia 2008; 46:1495-1504.

34 Castiglioni S, Pelati O, Zuffi M, Somalvico F, Marino L, Tentorio T, Franceschi M: The frontal assessment battery does not differentiate frontotemporal dementia from Alzheimer's disease. Dement Geriatr Cogn Disord 2006; 22:125-131.

35 Johnson DK, Morris JC, Galvin JE: Verbal and visuospatial deficits in dementia with Lewy bodies. Neurology 2005;65:1232-1238.

-36 Gustafson L, Erikson C, Warkentin S, Brun A, Englund E, Passant U: A factor analytic approach to symptom patterns in dementia. Int J Alzheimers Dis 2010;2011:632604.

-37 Hornberger M, Piguet 0, Graham AJ, Nestor PJ, Hodges JR: How preserved is episodic memory in behavioral variant frontotemporal dementia? Neurology 2010;74:472-479.

-38 Wicklund AH, Johnson N, Rademaker A, Weitner BB, Weintraub S: Word list versus story memory in Alzheimer disease and frontotemporal dementia. Alzheimer Dis Assoc Disord 2006;20:86-92.

-39 Pasquier F, Grymonprez L, Lebert F, Van der Linden M: Memory impairment differs in frontotemporal dementia and Alzheimer's disease. Neurocase 2001;7:161-171.

40 Hodges JR, Davies RR, Xuereb JH, Casey B, Broe M, Bak TH, Kril JJ, Halliday GM: Clinicopathological correlates in frontotemporal dementia. Ann Neurol 2004;56:399-406.

41 Braak H, Braak E: Neuropathological stageing of Alzheimer-related changes. Acta Neuropathol 1991;82:239259.

42 Rogers TT, Ivanoiu A, Patterson K, Hodges JR: Semantic memory in Alzheimer's disease and the frontotemporal dementias: a longitudinal study of 236 patients. Neuropsychology 2006;20:319-335.

-43 Laisney M, Matuszewski V, Mézenge F, Belliard S, de la Sayette V, Eustache F, Desgranges B: The underlying mechanisms of verbal fluency deficit in frontotemporal dementia and semantic dementia. J Neurol 2009;256: 1083-1094.

44 Salmon DP, Heindel WC, Lange KL: Differential decline in word generation from phonemic and semantic categories during the course of Alzheimer's disease: implications for the integrity of semantic memory. J Int Neuropsychol Soc 1999;5:692-703. 